\title{
First Wall Particle Flux Measurements by an F82H Permeation Probe in QUEST ${ }^{*}$
}

\author{
Haishan ZHOU, Yoshi HIROOKA ${ }^{1)}$, Hideki ZUSHI ${ }^{2)}$, Arseniy KUZMIN ${ }^{2)}$ and the QUEST group ${ }^{2)}$ \\ The Graduate University for Advanced Studies, Toki, Gifu 509-5292, Japan \\ 1) National Institute for Fusion Science, 322-6 Oroshi, Toki, Gifu 509-5292, Japan \\ ${ }^{2)}$ RIAM, Kyushu University, Kasuga, Fukuoka 816-8580, Japan
}

(Received 10 December 2013 / Accepted 7 March 2014)

\begin{abstract}
First wall particle flux measurements in the QUEST spherical tokamak have been conducted, using a permeation probe that employs a first wall candidate ferritic steel alloy F82H as the membrane and also SUS304 as a comparative reference membrane. Permeation measurements have been done during the conditioning steadystate discharges heated with $2.45 \mathrm{GHz}$ and $8.2 \mathrm{GHz}$ ECR. Diffusion and recombination coefficients measured in a laboratory-scale plasma device: VEHICLE-1 are used to interpret the results from the permeation probe measurements in QUEST. These permeation membranes have been analyzed with XPS to evaluate the effects of surface impurities.
\end{abstract}

(C) 2014 The Japan Society of Plasma Science and Nuclear Fusion Research

Keywords: plasma-driven permeation, QUEST, reduced activation ferritic steel, stainless steel, first wall, hydrogen plasma

DOI: $10.1585 /$ pfr.9.3405041

\section{Introduction}

In magnetic fusion power devices, hydrogenic particles will escape from the confinement region and then migrate through the first wall by plasma-driven permeation (PDP) [1-4]. Hydrogen isotopes (deuterium and tritium) flowing into the blanket by PDP will hinder the recovery of tritium and will probably necessitate isotope separation [2]. Tritium permeation through the first wall may raise reactor safety issues as well. It is highly desirable to perform the measurements of particle flow to the wall in fusion devices.

The incoming hydrogen flux to the first wall can be measured using a permeation probe $[5,6]$. For hydrogen PDP experiments, the hydrogen transport parameters such as diffusivity and recombination coefficient are essential to correctly interpret the measurement results. Unfortunately, the database of recombination coefficients for fusion materials has not well been compiled. The literature data span several orders of magnitude even for some of the most widely investigated metals such as stainless steel and nickel [7].

Reduced activation ferritic steels (RAFSs) such as $\mathrm{F} 82 \mathrm{H}$ are the candidate materials for the first wall of DEMO reactors. In our previous studies, hydrogen transport through $\mathrm{F} 82 \mathrm{H}$ has been systematically investigated using a steady-state laboratory-scale plasma device: VEHICLE-1 [8]. Permeation parameters including solubility, diffusivity and recombination coefficient have been

author's e-mail: zhou.haishan@LHD.nifs.ac.jp

*) This article is based on the presentation at the 23rd International Toki Conference (ITC23). measured in the temperature range from 150 to $520^{\circ} \mathrm{C}$ [3]. In the present work, membrane samples prepared in the same way as those used in VEHICLE-1 are installed on a permeation setup in the spherical tokamak QUEST, so that the surface conditions for all the samples are assumed to be the same and the hydrogen transport parameters taken in laboratory experiments can be used to analyze the measurement results in QUEST.

This paper presents the first results of the measurements using an F82H permeation probe in QUEST during conditioning steady-state discharges. The effects of surface impurities on PDP behavior are also discussed.

\section{Experimental}

QUEST [9] is a medium size spherical tokamak with a full metal chamber made of stainless steel. The chamber radius and height are $\sim 1.4 \mathrm{~m}$ and $\sim 2.8 \mathrm{~m}$, respectively. The total surface area of the chamber wall is $\sim 35.5 \mathrm{~m}^{2}$ and the volume is $\sim 13 \mathrm{~m}^{3}$ including the extension ports. Outboard limiters made of tungsten are installed on the outside walls at a major radius of $R=1.35 \mathrm{~m}$. Hydrogen plasmas are produced using electron cyclotron resonance with three kinds of RF sources: $2.45 \mathrm{GHz}, 8.2 \mathrm{GHz}$ and $28 \mathrm{GHz}$. In the present work, hydrogen permeation experiments have been performed for the low temperature, low density slab plasmas in discharge cleaning experiments using the $2.45 \mathrm{GHz}$ and the $8.2 \mathrm{GHz}$ sources. The slab plasma means plasmas produced in the electron cyclotron resonance region without poloidal field (i.e., no closed flux surfaces) [6].

Shown in Fig. 1 are a schematic diagram of the probe position and the PDP setup in QUEST. The permeation 
(a)

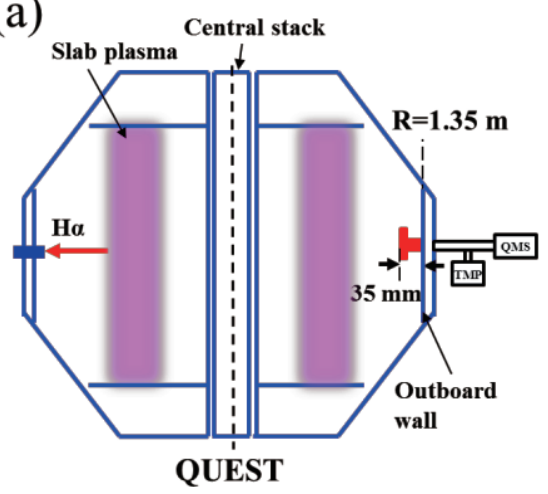

(b)

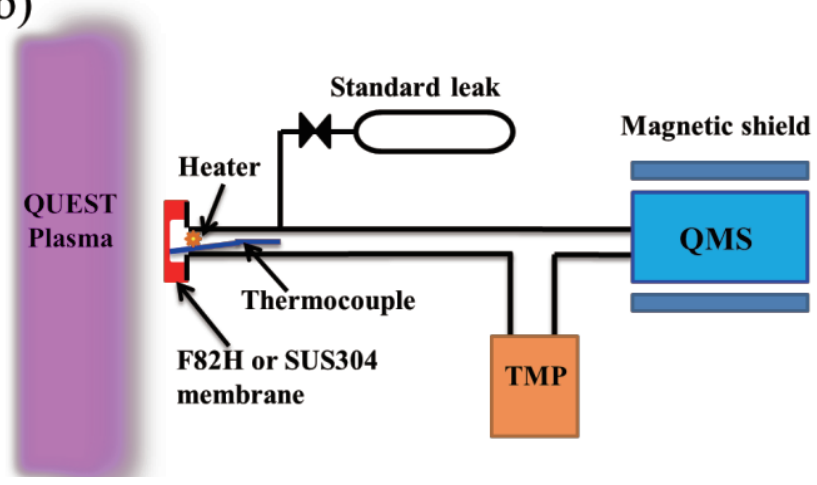

Fig. 1 Schematic diagrams of (a) the permeation probe setup in QUEST and (b) the details of the permeation flux measurement system.

probe has been installed near the mid-plane and the permeation membrane is $35 \mathrm{~mm}$ away from the outboard wall in the radial direction (Fig. 1 (a)). A resistive heater is set behind the sample so that the membrane temperature can be kept in a range of $240-300^{\circ} \mathrm{C}$. The temperature is measured by a thermocouple attached to the downstream surface. The hydrogen partial pressure is measured by a quadrupole mass spectrometer (QMS), which has been calibrated by a hydrogen standard leak, as shown in Fig. 1 (b).

Samples made of F82H and SUS304 are prepared in the same dimensions as those commercially available conflat flanges with an outer diameter of $34 \mathrm{~mm}$, except that a circular area of $\sim 16 \mathrm{~mm}$ in diameter inside the knife-edge is machined down to thicknesses of 0.14 to $0.5 \mathrm{~mm}$. The samples are cut from the F82H plates used in the JFT-2 M tokamak at JAERI (now JAEA) [10]. The stainless steel sample is used as a comparative reference.

After the permeation experiments, the membrane surfaces are analyzed with energy dispersive X-ray spectroscopy (EDX) and X-ray photoelectron spectroscopy (XPS). For XPS analysis, a $4 \mathrm{kV} \mathrm{Ar}^{+}$gun is used to etch the sample surfaces so that the depth profile can be obtained.

\section{Results and Discussion}

\subsection{PDP through F82H and SUS304 mem- branes in QUEST}

Figure 2 shows the hydrogen PDP data through a $0.2 \mathrm{~mm}$ thick F82H membrane exposed to a wall conditioning $900 \mathrm{~s}$ discharge. The plasma is produced by the $2.45 \mathrm{GHz}$ RF system with an input power of $7.5 \mathrm{~kW}$ and the toroidal filed coil current is kept at $17 \mathrm{kA}$. The steady-state permeation flux for the $\mathrm{F} 82 \mathrm{H}$ membrane has been measured to be $\sim 2.4 \times 10^{13} \mathrm{H} \cdot \mathrm{cm}^{-2} \cdot \mathrm{s}^{-1}$ at $\sim 270^{\circ} \mathrm{C}$.

Shown in Fig. 3 is a comparison of PDP behavior through $0.2 \mathrm{~mm}$ thick F82H and $0.14 \mathrm{~mm}$ thick SUS304 membranes. It can be seen that the permeation flux through the F82H membrane can reach steady state within $100 \mathrm{~s}$. For SUS304, in contrast the permeation flux keeps increasing during the discharge, not reaching the steady state.

Figure 4 shows the results of PDP through the SUS304

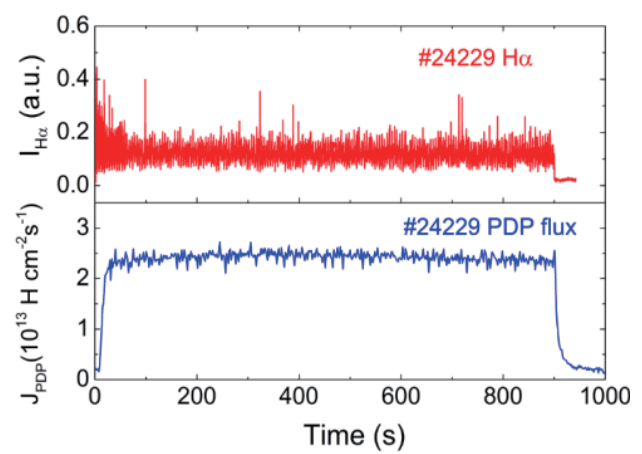

Fig. 2 Plasma-driven permeation through a $0.2 \mathrm{~mm}$ thick $\mathrm{F} 82 \mathrm{H}$ membrane at a temperature around $270^{\circ} \mathrm{C}$.

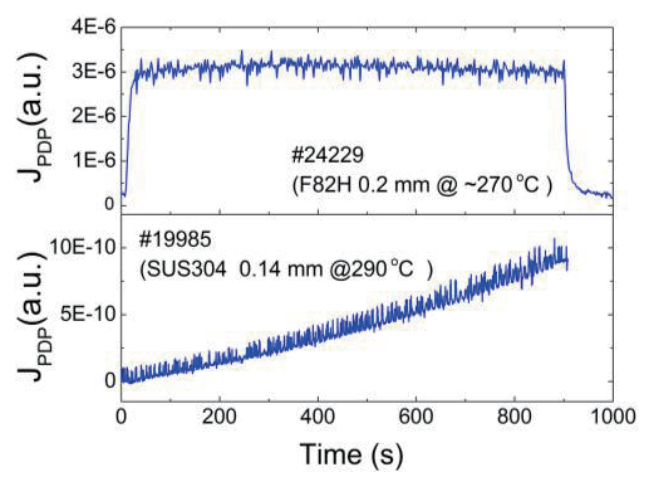

Fig. 3 Comparison of PDP through F82H and SUS304 membranes in $900 \mathrm{~s}$ discharges.

membrane in four continual discharges. The total exposure time is about $3600 \mathrm{~s}$, but the permeation flux cannot reach steady state. These results are in consistent with the experimental observation for the F82H and SUS304 membranes in VEHICLE-1 [1].

In the steady-state plasma-driven permeation model, three regimes are considered [11]: the diffusion-diffusion (DD) limited regime, the recombination-diffusion (RD) limited regime and the recombination-recombination (RR) limited regime. The rate controlling process can be characterized by the dimensionless parameter [11]: $W=$ 


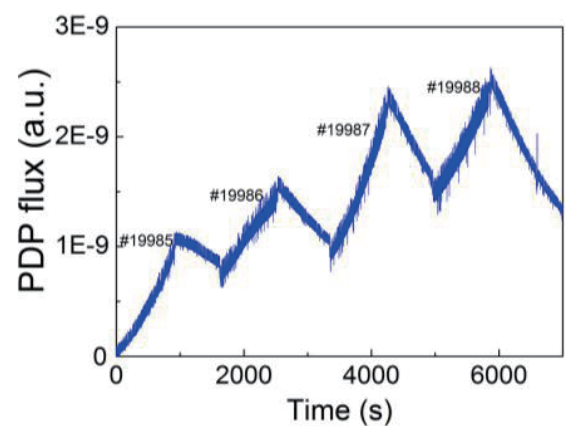

Fig. 4 Plasma-driven permeation through SUS304 in four continual $900 \mathrm{~s}$ shots at $290^{\circ} \mathrm{C}$.

Table 1 Calculation results of $W$ at various temperatures.

\begin{tabular}{|c|c|c|c|}
\hline $\begin{array}{c}\text { Temperature } \\
\left({ }^{\circ} \mathrm{C}\right)\end{array}$ & $\begin{array}{c}D \\
\left(\mathrm{~cm}^{2} \cdot \mathrm{s}^{-1}\right)\end{array}$ & $\begin{array}{c}K_{\mathrm{r}} \\
\left(\mathrm{cm}^{4} \cdot \mathrm{s}^{-1}\right)\end{array}$ & $W$ \\
\hline 220 & $2.8 \times 10^{-5}$ & $1.2 \times 10^{-15}$ & 0.122 \\
\hline 290 & $4.2 \times 10^{-5}$ & $1.6 \times 10^{-16}$ & 0.030 \\
\hline
\end{tabular}

$R\left(J_{0} K_{\mathrm{r}}\right)^{1 / 2} / D$, where $R$ is the implantation range, $J_{0}$ is the implantation flux, $K_{\mathrm{r}}$ is the recombination coefficient for the front surface and $D$ is the diffusion coefficient. The overall hydrogen transport is controlled by the slowest process and the parameter $W$ may be regarded as a competition between diffusion and recombination. The implantation depth $R$ in iron is only several $\mathrm{nm}$ for hydrogen ions and neutral atoms [12]. The diffusion and recombination coefficients have been calculated from the VEHICLE-1 data to be [3]:

$$
D=7.5 \times 10^{-4} \exp \left(\frac{-0.14[\mathrm{eV}]}{k T}\right)\left[\mathrm{cm}^{2} \cdot \mathrm{s}^{-1}\right]
$$

and

$$
K_{\mathrm{r}}=1.3 \times 10^{-22} \exp \left(\frac{0.68[\mathrm{eV}]}{k T}\right)\left[\mathrm{cm}^{4} \cdot \mathrm{s}^{-1}\right]
$$

respectively. Table 1 shows the calculation results of $W$, which indicates that PDP through the F82H is in the RDregime $(R / L<W<1$, where $L$ is the membrane thickness), i.e., recombination-limited at the front surface and diffusion-limited inside the bulk.

Using Eq. (1) and the diffusivity data for stainless steel [13], the diffusion distances have been estimated to be $1.9 \mathrm{~mm}$ for $\mathrm{F} 82 \mathrm{H}$ and $0.019 \mathrm{~mm}$ for SUS304 in $900 \mathrm{~s}$ at $290^{\circ} \mathrm{C}$, which can explain their different permeation behavior shown in Fig. 3. The calculation result is also in agreement with the trend that hydrogen can transport faster in bcc metals (e.g., ferritic steels) than in fcc metals (e.g., SUS304) [14].

\subsection{Effect of plasma heating power}

Shown in Fig. 5 are the $\mathrm{H} \alpha$ intensity and permeation flux data for two $900 \mathrm{~s}$ discharges with different heating methods. The long-pulse plasmas are maintained by the $2.45 \mathrm{GHz}$ RF source with a power of $4 \mathrm{~kW}$. For shot

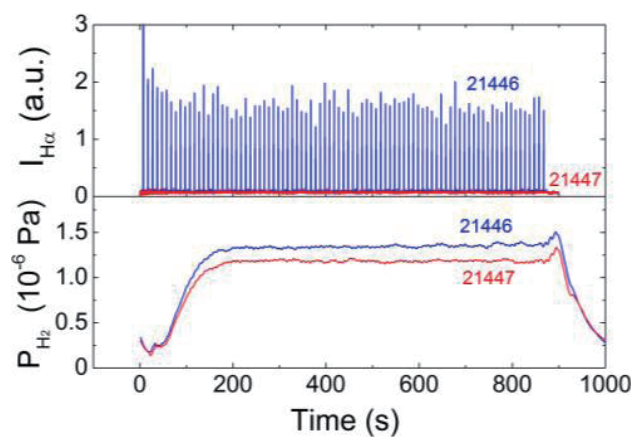

Fig. 5 PDP through F82H membrane w/ and w/o the $8.2 \mathrm{GHz}$ RF plasma heating source.

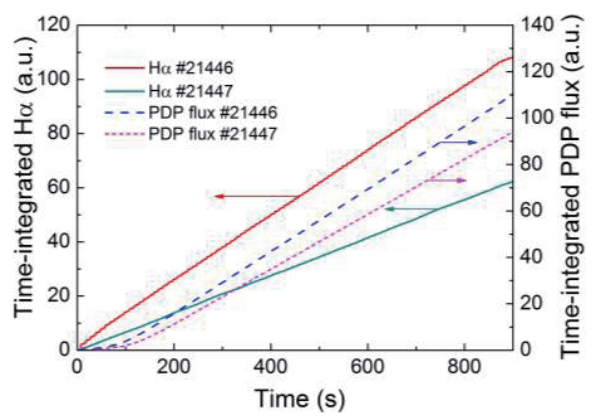

Fig. 6 The time-integrated $\mathrm{H} \alpha$ intensities and PDP fluxes for the \#21446 and \#21447 shots.

\#21446, additional $8.4 \mathrm{GHz}$ ECR heating $(25 \mathrm{~kW}, 0.4 \mathrm{~s}$ width) is conducted throughout the discharge with a frequency of $0.1 \mathrm{~Hz}$. Permeation flux measurements show that without $8.2 \mathrm{GHz}$ RF heating, the steady-state PDP flux decreases by $\sim 13 \%$ for F $82 \mathrm{H}$. Due to the lack of plasma temperature and density data, the time-integrated $\mathrm{H} \alpha$ intensity $\left(Q_{\mathrm{H} \alpha}\right)$ is used as a measure to estimate the particle flux to the wall $\left(J_{0}\right)[6]$.

Figure 6 shows the time-integrated $\mathrm{H} \alpha$ intensities and the PDP fluxes. At $t=900 \mathrm{~s}$, where $t$ is the time, the value of $\left(Q_{\mathrm{H} \alpha}\right)^{1 / 2}$ for shot \#21446 is higher than that of shot \#21447 by a factor of $\sim 1.41$, while the permeation flux $\left(J_{+}\right)$ratio of the two discharges is $\sim 1.13$. Data roughly agree with the theoretical prediction for the permeation flux $J_{+}\left[\right.$atom $\left.\cdot \mathrm{cm}^{2} \cdot \mathrm{s}^{-1}\right]$ when plasma-driven permeation takes place in the RD-regime:

$$
J_{+}=\frac{D}{L} \sqrt{\frac{J_{0}}{K_{\mathrm{r}}}}
$$

i.e., the steady-state permeation flux is proportional to the square root of the implantation flux.

\subsection{Diffusivity measurements for $\mathrm{F82H}$ from the PDP data taken in QUEST}

The diffusivity for hydrogen through a metal can be obtained either by fitting the transient permeation curve or by measuring the time lag $t_{1}=L^{2} / 6 D$ [3]. Shown in Fig. 7 are the effective diffusivity data measured for $\mathrm{F} 82 \mathrm{H}$ in the 


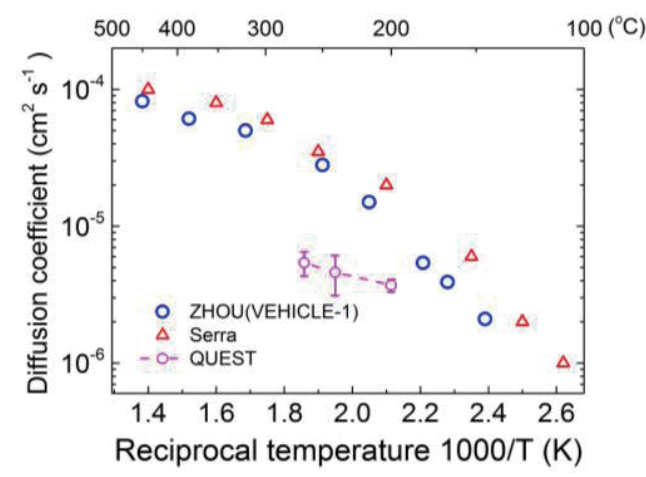

Fig. 7 Effective diffusivity measurements for F82H in QUEST. The previous VEHICLE-1 data [3] and Serra' s data [15] are shown for a comparison.

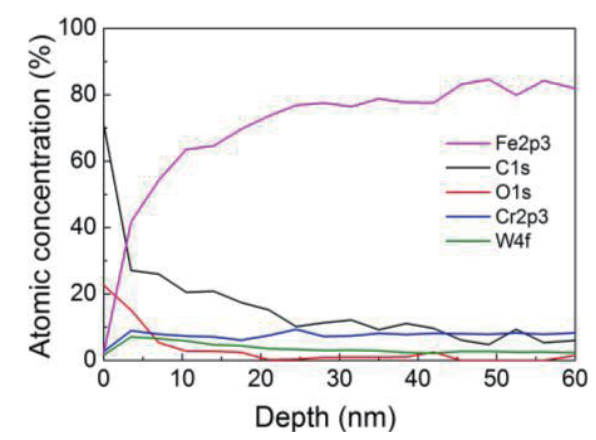

Fig. 8 Surface composition of an F82H membrane after the PDP experiments in QUEST.

PDP experiments in QUEST. The previous VEHICLE-1 data [3] and Serra's data [15] are shown for comparison. It has been found that the diffusion coefficients estimated from the QUEST data are lower by a factor of 3 to 4 than those taken in VEHICLE-1, although the sample membranes are essentially the same.

Hydrogen PDP has often been observed to be enhanced by surface contaminations because recombination release is suppressed by the presence of impurities. However, it is also true that if the contaminated layer becomes thick enough to act as a second layer for diffusion $[4,16]$. One possible reason for the lower measured diffusivity is that the membrane surface is contaminated during the PDP experiments in QUEST. Figure 8 shows the surface analysis using X-ray photoelectron spectroscopy (XPS) for an F82H membrane after the PDP experiments in QUEST. Impurities such as carbon, tungsten and oxygen have been detected on the membrane surface. Carbon deposition should be made during plasma exposures because contam- inations from air exposure can only affect the first several $\mathrm{nm}$ of the sample surface. Tungsten impurity should come from erosion and deposition of the plasma-facing components [17].

\section{Conclusion}

Plasma-driven permeation (PDP) measurements have been conducted in the QUEST spherical tokamak and a steady state laboratory-scale plasma device: VEHICLE1 for $\mathrm{F} 82 \mathrm{H}$ and SUS304. A much shorter PDP breakthrough time and higher steady-state permeation flux have been found for F82H than SUS304. The F82H permeation probe shows a good sensitivity to the variation of plasma parameters. However, the permeation flux measurements may be affected by the surface impurities accumulated in plasma discharges, which warrants further investigation.

\section{Acknowledgement}

The author would like to thank Mr. J. Yagyu, Dr. M. Sato, Dr. T. Nakano and Dr. A. Sakasai of JAEA, Naka for their material preparation.

[1] H. Zhou et al., Fusion Sci. Technol. 63, 1T, 361 (2013).

[2] Y. Hirooka et al., Fusion Sci. Technol. 64, 345 (2013).

[3] H. Zhou et al., "Gas- and plasma-driven hydrogen permeation through a reduced activation ferritic steel alloy F82H", Paper presented at the 16th International Conference on Fusion Reactor Materials, Beijing, China, Oct. 20th-26th, 2013.

[4] Y. Hirooka et al., "Bi-directional hydrogen isotopes permeation through the first wall of a magnetic fusion demo reactor", Paper presented at the 16th International Conference on Fusion Reactor Materials, Beijing, China, Oct. 20th26th, 2013.

[5] J. Winter et al., J. Nucl. Mater. 111 \& 112, 243 (1982).

[6] S.K. Sharma et al., J. Nucl. Mater. 420, 83 (2012).

[7] O.V. Ogorodnikova et al., Fusion Eng. Des. 49-50, 921 (2000).

[8] Y. Hirooka et al., J. Nucl. Mater. 337-339, 585 (2005).

[9] K. Hanada et al., Plasma Fusion Res. 5, S1007 (2010).

[10] H. Kimura et al., Fusion Eng. Des. 56-57, 837 (2001).

[11] B.L. Doyle, J. Nucl. Mater. 111\&112, 628 (1982).

[12] J.F. Ziegler et al., Nucl. Instrum. Methods B12, 1818 (2010).

[13] Möller and J. Roth, Physics of Plasma-Wall Interaction in Controlled Fusion Devices (Plenum Press, New York, 1986) p. 439-494.

[14] J. Völkl and G. Alefeld, Hydrogen in metals I (SpringerVerlag, Berlin, 1978) p. 321-348.

[15] E. Serra et al., J. Nucl. Mater. 245, 108 (1997).

[16] Y. Hatano et al., Vac. Sci. Technol. A16, 2078 (1998).

[17] S.K. Sharma et al., Fusion Eng. Des. 87, 77 (2012). 